\title{
Contrast-Enhanced CT-Guided Core Biopsy of Retroperitoneal Masses
}

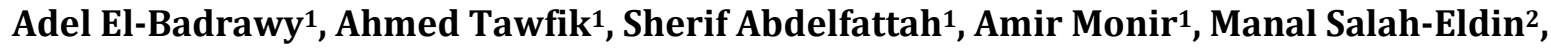 \\ Emad Eldin Azmy3, Dina El-Tantawy ${ }^{4}$, Amro El-Karef ${ }^{4}$ \\ ${ }^{1}$ Radiology Department, Mansoura Faculty of Medicine, Mansoura, Egypt \\ ${ }^{2}$ Medical Oncology Department, Mansoura Faculty of Medicine, Mansoura, Egypt \\ ${ }^{3}$ Clinical Hematology Department, Mansoura Faculty of Medicine, Mansoura, Egypt \\ ${ }^{4}$ Pathology Department, Mansoura Faculty of Medicine, Mansoura, Egypt \\ Email: adelelbadrawy@hotmail.com
}

Received 15 November 2013; revised 15 December 2013; accepted 30 December 2013

Copyright (C) 2014 by authors and Scientific Research Publishing Inc.

This work is licensed under the Creative Commons Attribution International License (CC BY).

http://creativecommons.org/licenses/by/4.0/

(c) (i) Open Access

\begin{abstract}
Objective: To evaluate the efficacy, feasibility and safety of contrast-enhanced CT-guided core biopsy of retroperitoneal masses. Materials and Methods: Contrast-enhanced CT-guided biopsy of retroperitoneal masses was performed in 26 patients. Histopathological diagnosis was obtained and accuracy was calculated. Two blinded radiologists determined feasibility of biopsy procedures on routine CT-guidance, and compared accessibility of target lesions using contrast-enhanced and non-contrast-enhanced CT. Results: Satisfactory samples were obtained in all 26 patients and pathological diagnosis was made in 24 . Accuracy was $95.5 \%$. Routine CT-guided biopsy punctures were not satisfying in 4/26 patients, and contrast-enhanced CT scans rendered the target lesions more accessible in 16 patients. Conclusion: Contrast-enhanced CT-guided biopsy increases the feasibility of biopsy of retroperitoneal masses.
\end{abstract}

\section{Keywords}

Contrast-Enhanced; CT-Guided; Biopsy; Retroperitoneal Masses

\section{Introduction}

Computed Tomography (CT)-guided needle biopsy of retroperitoneal masses is widely accepted as an effective and safe procedure to reach pathologic diagnosis in many clinical settings [1] [2]. In recent years, very high diagnostic accuracy of CT-guided needle biopsy for definitive histological diagnosis of lymphoma and other re- 
troperitoneal masses has been reported, thanks to technical advances in both the biopsy as well as the histological diagnostic procedures [3] [4]. However, in many instances, a safe approach to obtaining biopsy from the retroperitoneum will not be secured due to interruption of the planned puncture path by major vessels or ureters adjacent to the target lesion, especially with small lesions. In these cases, percutaneous needle biopsy will be abandoned and the patient will be referred to surgical biopsy.

Until the time of performing this study, all CT-guided biopsy reports from retroperitoneal masses described the use of non-contrast enhanced CT to guide the introduction of the needle into the target lesion. We postulated that simultaneous intravenous contrast administration at the time of performing biopsy will increase the feasibility of CT-guided biopsies of retroperitoneal masses. In this study we prospectively evaluated the efficacy, feasibility and safety of contrast-enhanced CT-guided core biopsy of retroperitoneal masses.

\section{Materials and Methods}

This study included 26 patients with retroperitoneal masses that were referred for percutaneous imaging-guided biopsy. The patients were 21 men and 5 women (age ranged between 21 - 64 years; mean age 42 years). The coagulation parameters and platelet counts were obtained in all patients to exclude any bleeding diathesis. All patients provided written informed consent for the procedure. The study was carried out from November 2011 to September 2012 at Oncology Center, Mansoura University. Institutional review board approval was obtained.

Previous contrast-enhanced CT scans were reviewed by the interventionist performing the biopsy before the procedure to determine the exact tumor location, the degree of vascularity and the presence of necrosis and then plan the puncture pathway.

The biopsy procedures were performed on a 64-MDCT scanner (Brilliance 64-Philips). A posterior paraspinal approach was feasible in all patients. First, a guide spinal needle of 25G was placed for local anesthesia using CT-fluoroscopy without IV contrast injection. Next, the fine spinal needle was removed and tru-cut needle biopsy was placed approximately near the lesion away from the expected site of aorta, IVC, ureter and renal vessels. Then $50 \mathrm{ml}$ of non-ionic contrast medium (ioversol, Optiray 350) at a flow rate of $3-4 \mathrm{ml} / \mathrm{sec}$ were administered intravenously. Imaging was performed at late arterial phase (30 - 40 seconds delay). The CT acquisition parameters were $200 \mathrm{mAs}, 120 \mathrm{kVp}, 512 \times 512$ matrix, 1.172 pitch, $64 \times 0.625 \mathrm{~mm}$ section collimation, $4 \mathrm{~mm}$ slice thickness. In lesions at or below the level of renal pelvis, delayed contrast-enhanced CT scan was done for delineation of the ureters.

Using a manually advanced, spring-loaded automatic coaxial biopsy needle system 4 - 6 co-axial biopsy cores were taken. An 18G needle was used in 14 cases and a 16G needle was done in 12 cases. The size of the needle was determined by the interventionist at the time of biopsy based on the site and volume of disease and estimated difficulty and risk of the procedure. The biopsy procedure lasted 20 - 35 minutes and was generally well tolerated in all patients. Patients were observed for 1 - 3 hours after the procedure to ensure their hemodynamic stability and to monitor their respiratory status.

The lesion size and location, needle path, number of needle penetrations, reasons for failure, biopsy results, and complications were all recorded. All biopsy specimens were subjected to pathological evaluation. Diagnosis was validated with further multidisciplinary review, subsequent surgery, and response to specific chemotherapy.

After the last procedure in the study was finished, the CT scans were reviewed in consensus by 2 interventionists other than the one who performed the biopsies. The 2 radiologists, who had more than 5 years of experience in CT-guided biopsies, were first blinded to the purpose and results of the study and were asked to plan a biopsy puncture as usual (previous contrast-enhanced CT as road map, biopsy guided by non-contrast-enhanced $\mathrm{CT}$ ) and to determine, in consensus, whether a safe access for every patient was guaranteed or not. Afterwards, the aim of the study was explained to them and they were asked to determine whether the use of contrast-enhanced CT for biopsy guidance increased the accessibility of target lesions and level of confidence or not.

\section{Results}

No major complications were observed at the time or after the procedure, defined as those requiring surgery or blood transfusion. Minor complications were seen in 7 patients. Three patients complained of sciatic pain during the procedure but, on changing the direction of the needle, the pain was relieved. The remaining 4 patients had transient pain at the puncture site that eventually subsided without medications.

Core tissue was obtained in all 26 patients. Pathologic diagnosis was possible for 24 of 26 patients (92.3\%). In 
the remaining two patients, the sample was necrotic even with repeated biopsy.

The sensitivity, specificity, and accuracy for all biopsies were 94.5, 100.0, and 95.5\%. There were no significant differences between 16g and 18G needle biopsy. The masses were non-Hodgkin lymphoma ( $\mathrm{n}=7$ ) (Figure 1), Hodgkin disease $(n=5)$ (Figure 2), metastatic adenocarcinoma $(n=4)$ (Figure 3), paraganglioma $(n=3)$, neurofibroma $(n=2)$, retroperitoneal fibrosis $(n=2)$, hyalinized tissue after chemotherapy for NHL $(n=1)$ and necrotic tumor $(\mathrm{n}=2)$ (Table 1$)$.

As regard blinded evaluation by the 2 interventional radiologists, routine CT-guided biopsy (previous contrast-enhanced CT as road map, biopsy guided by non-contrast-enhanced CT) was judged not feasible in 4 out of 26 patients. Target leiosns were judged more accessible on contrast-enhanced CT than non-contrast-enhanced CT scans in 16 out of the remaining 22 patients.

\section{Discussion}

The optimal management of patients with retroperitoneal masses depends on obtaining specific pathologic diagnosis. Percutaneous imaging-guided needle biopsy is now an established technique that has replaced open surgical biopsy in most of the cases because it is less invasive, less expensive and reduces hospital stay. The use

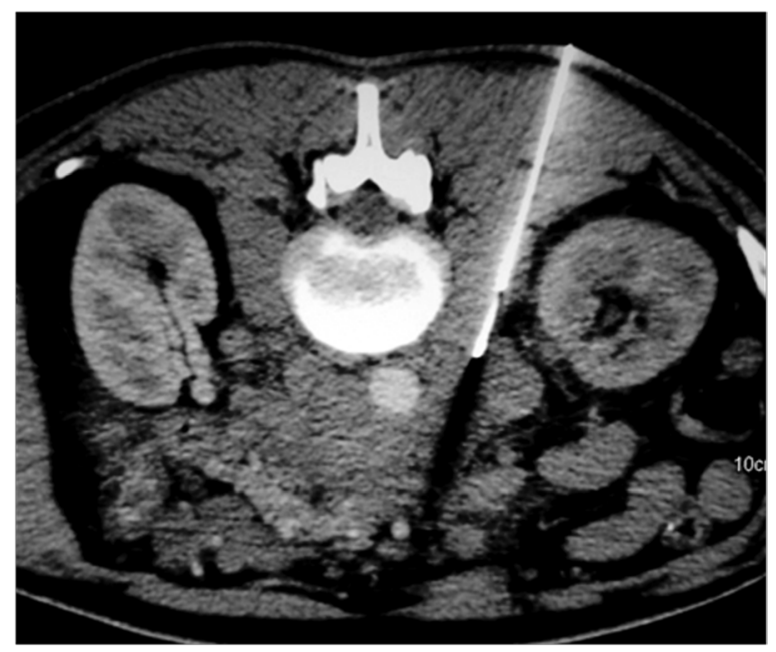

Figure 1. 52-year-old man presented with abdominal lymphadenopathy. Axial contrast-enhanced CT image at the time of biopsy needle introduction shows the needle passing safely to the left side of the aorta. Pathologically proved non-Hodgkin lymphoma.

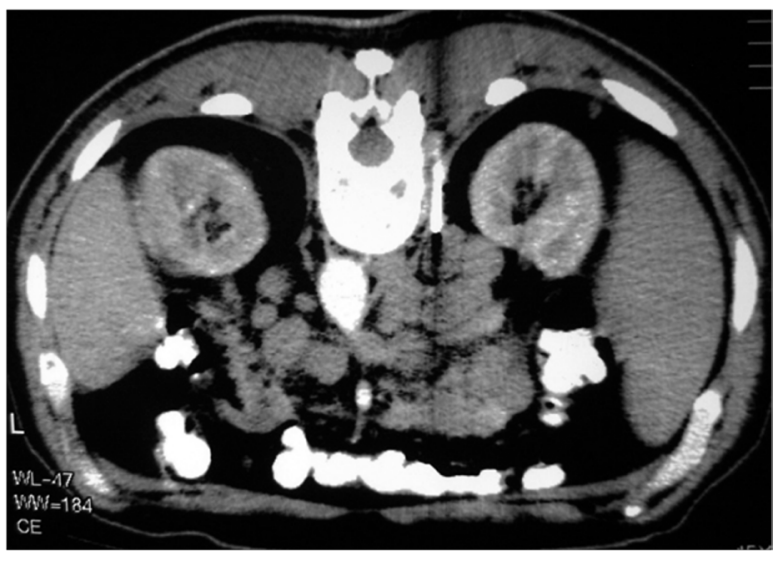

(a)

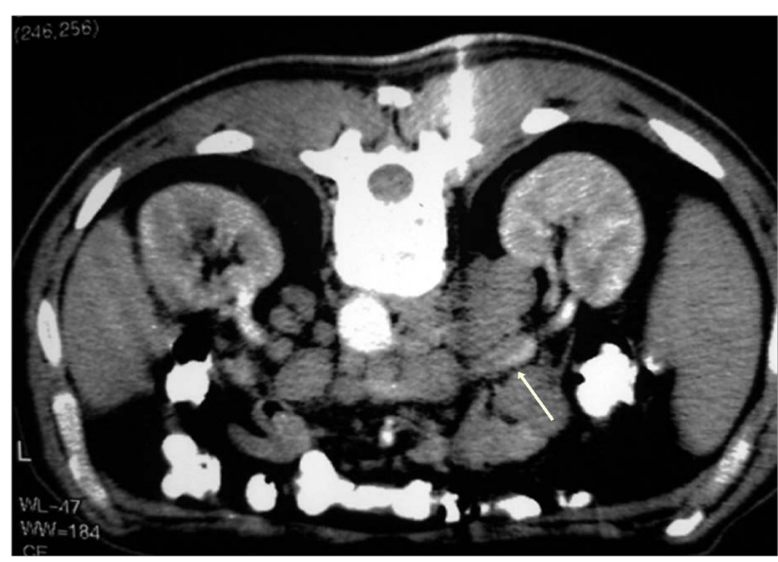

(b)

Figure 2. 58-year-old man presented with abdominal lymphadenopathy. (a) and (b) Axial contrast-enhanced CT images at the time of biopsy needle introduction showing the needle passing safely to the right side of the aorta and away from IVC. The IVC is displaced anterior by lymphadenopathy. Pathologically proved Hodgkin disease. 


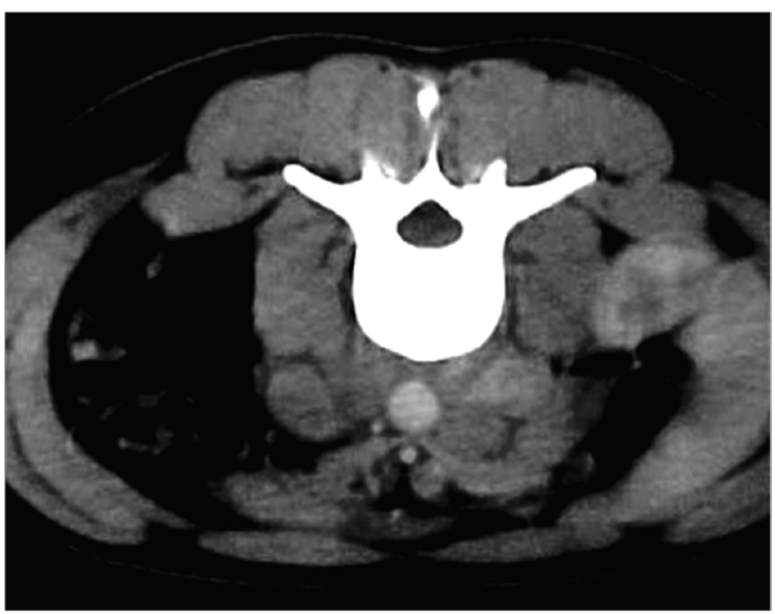

(a)

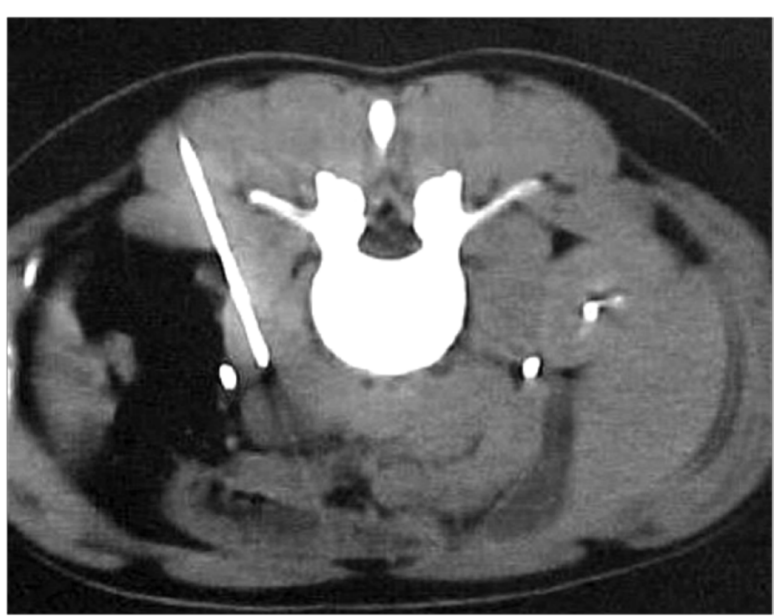

(b)

Figure 3. 45-year-old man presented with abdominal lymphadenopathy. (a) Contrast-enhanced CT image before biopsy needle introduction shows the lymphadenopathy and the aorta and IVC. (b) Delayed CT image at the time of biopsy needle introduction shows the ureters and the needle passing safely between left ureter and aorta. Pathologically proved metastatic adenocarcinoma.

Table 1. Pathological diagnosis of 26 patients with retroperitoneal masses.

\begin{tabular}{cc}
\hline Final diagnosis & Number of patients \\
\hline Non-Hodgkin lymphoma & $\mathbf{7}$ \\
Hodgkin disease & $\mathbf{5}$ \\
Metastatic adenocarcinoma & $\mathbf{4}$ \\
Paraganglioma & 3 \\
Neurofibroma & $\mathbf{2}$ \\
Retro-peritoneal fibrosis. & $\mathbf{2}$ \\
Hayalinized tissue after chemotherapy for NHL & $\mathbf{1}$ \\
Necrotic tumor & $\mathbf{2}$ \\
Total & $\mathbf{2 6}$ \\
\hline
\end{tabular}

NHL: Non-Hodgkin lymphoma.

surgical biopsy in most of the cases because it is less invasive, less expensive and reduces hospital stay. The use of cross-sectional abdominal imaging for diagnostic purposes is continuously increasing, and more lesions with smaller sizes are being detected. The safety and efficacy of percutaneous CT-guided needle biopsy of retroperi-toneal masses is well documented [5]-[7]. Many authorities prefer CT- to ultrasound-guidance for retroperitoneal masses because of its wide field of view and clear visualization [7] [8]. With CT-guidance, a posterior approach is possible which caries lower risk of bleeding and bowel perforation compared to the anterior approach provided by ultrasound-guidance [8]. The posterior approach also decreases breath motion and pain.

Retroperitoneal masses usually encase or contact the aorta, inferior vena cava, kidneys, ureters and/or other structures. In several cases, a safe puncture pathway will be difficult to plan on the basis of a previous contrast-enhanced CT scan and the procedure will be judged as inaccessible. The described method of contrast-enhanced CT-guidance helps to identify and avoid these major structures adjacent to the target biopsy site. This study demonstrated that contrast-enhanced CT-guided percutaneous biopsy of retroperitoneal masses is safe and accurate, resulting in a tissue-specific pathological diagnosis in $92.3 \%$ of patients. Biopsy needles of 18 and 16 gauge were used with no complications of haemorrhage, bowel perforation or organ injuries. In 4 out of 26 patients, biopsy would have not been performed if non-contrast-enhanced CT was used, but the use of contrast-enhanced CT changed the decision. In 16 out of 22 patients, contrast-enhanced CT was judged to increase accessibility of the puncture path and the level of confidence of the interventionist.

Collins et al. investigated the simultaneous use of intravenous contrast during CT-guided needle biopsy of 
pancreatic carcinomas encasing the celiac or superior mesenteric arteries [9]. Their results were excellent (100\% success) although the number of patients was small (11 patients). Meier-Meitinger et al. reported that CT-guided biopsies of pancreatic lesions with IV contrast administration during needle placement were more accurate than biopsies performed after contrast-enhanced planning CT [10].

At our institution, on-site instant cytological review is not routinely available, and hence biopsy of peritoneal disease is preferred to FNAC. In addition, core biopsy allows for the architectural features of the lesion to be determined and provides more tissue for immune-histochemical analysis [4]. Core needle biopsies are thought of as superior to fine needle aspiration biopsies for accurate subtyping of lymphomas which is important to determine the treatment modalities [4] [8]. Our results for diagnostic accuracy of core needle biopsy are similar to those reported in literature [7] [8].

There was no difference between the accuracy of 16 gauge and 18 gauge needles in our study. This is in agreement with de Kerviler et al. [11], who reported no significant differences between needle sizes ranging from 20- up to14-gauge in the diagnostic accuracy of lymphoma. On careful review of the literature, however, we noted that there is no universal agreement whether different needle sizes do affect diagnostic yield or not. For example, in biopsies of focal hepatic lesions, one study compared the results of biopsy in 268 patients and reported similar results for 18- and 14-gauge needles [12]. On the other hand, another study on 163 patients reported a significantly higher diagnostic yield for 16-gauge compared to 18-gauge needles [13]. In our opinion, the most important factor for procedural success is obtaining sufficient sample volume. Another main important factor is the experience of the pathologist.

The main limitation of our study is the relatively small size of our sample. A further study is recommended including a larger series with randomized comparison between contrast-enhanced and non-contrast-enhanced CT-guided biopsy of retroperitoneal masses.

\section{Conclusion}

Our results demonstrated that the simultaneous use of IV contrast administration at the time of CT-guided biopsy increases the accessibility of retroperitoneal masses and the level of confidence of interventionists.

\section{References}

[1] Ben-Yehuda, D., Polliack, A., Okon, E., et al. (1996) Image-Guided Core-Needle Biopsy in Malignant Lymphoma: Experience with 100 Patients That Suggests the Technique Is Reliable. Journal of Clinical Oncology, 14, 2431-2434.

[2] Guimaraes, A.C., Chapchap, P., de Camargo, B., et al. (2003) Computed Tomography-Guided Needle Biopsies in Pediatric Oncology. Journal of Pediatric Surgery, 38, 1066-1068. http://dx.doi.org/10.1016/S0022-3468(03)00194-5

[3] Balestreri, L., Morassut, S., Bernardi, D., et al. (2005) Efficacy of CT-Guided Percutaneous Needle Biopsy in the Diagnosis of Malignant Lymphoma at First Presentation. Clinical Imaging, 29, 123-127. http://dx.doi.org/10.1016/j.clinimag.2004.10.002

[4] Demharter, J., Muller, P., Wagner, T., et al. (2001) Percutaneous Core-Needle Biopsy of Enlarged Lymph Nodes in the Diagnosis and Subclassification of Malignant Lymphomas. European Radiology, 11, 276-283. http://dx.doi.org/10.1007/s003300000540

[5] Welch, T.J., Sheedy II, P.F., Johnson, C.D., et al. (1989) CT-Guided Biopsy: Prospective Analysis of 1,000 Procedures. Radiology, 171, 493-496.

[6] Chojniak, R., Isberner, R.K., Viana, L.M., et al. (2006) Computed Tomography Guided Needle Biopsy: Experience from 1,300 Procedures. Sao Paulo Medical Journal, 124, 10-14. http://dx.doi.org/10.1590/S1516-31802006000100003

[7] Tomozawa, Y., Inaba, Y., Yamaura, H., et al. (2011) Clinical Value of CT-Guided Needle Biopsy for Retroperitoneal Lesions. Korean Journal of Radiology, 12, 351-357. http://dx.doi.org/10.3348/kjr.2011.12.3.351

[8] Stattaus, J., Kalkmann, J., Kuehl, H., et al. (2008) Diagnostic Yield of Computed Tomography Guided Coaxial Core Biopsy of Undetermined Masses in the Free Retroperitoneal Space: Single-Center Experience. CardioVascular and Interventional Radiology, 31, 919-925. http://dx.doi.org/10.1007/s00270-008-9317-5

[9] Collins, J.M., Kriegshauser, J.S. and Leslie, K.O. (2009) CT-Guided Biopsy of Perivascular Tumor Encasement Using Simultaneous IV Contrast Enhancement. American Journal of Roentgenology, 193, W283-W287. http://dx.doi.org/10.2214/AJR.08.1935

[10] Meier-Meitinger, M., Anders, K., Alibek, S., et al. (2009) CT-Guided Biopsies of Pancreatic Lesions: Impact of Contrast Application Prior to versus Following Needle Placement. Academic Radiology, 16, 1386-1392.

http://dx.doi.org/10.1016/j.acra.2009.04.013 
[11] de Kerviler, E., Guermazi, A., Zagdanski, A.M., et al. (2000) Image-Guided Core-Needle Biopsy in Patients with Suspected or Recurrent Lymphomas. Cancer, 89, 647-652.

http://dx.doi.org/10.1002/1097-0142(20000801)89:3<647::AID-CNCR21>3.0.CO;2-R

[12] Haage, P., Piroth, W., Staatz, G., et al. (1999) CT-Guided Percutaneous Biopsies for the Classification of Focal Liver Lesions: A Comparison between $14 \mathrm{G}$ and $18 \mathrm{G}$ Puncture Biopsy Needles. Rofo, 171, 44-48. http://dx.doi.org/10.1055/s-1999-9895

[13] Stattaus, J., Kühl, H., Hauth, E.A., et al. (2007) Liver Biopsy under Guidance of Multislice Computed Tomography: Comparison of 16G and 18G Biopsy Needles. Radiologe, 47, 430-438. http://dx.doi.org/10.1007/s00117-005-1336-5

\section{Abbreviations}

CT: computed tomography

MDCT: multi-detector computed tomography

NHL: non-Hodgkin's lymphoma

FNAC: fine needle aspiration cytology 